\title{
Pulling a Chain's Leg: The Pullout Dynamics of Entangled Chains
}

\author{
Raphael Blumenfeld* \\ Polymers and Colloids, Cavendish Laboratory, Madingley Road, Cambridge CB3 OHE, U.K. \\ Received J uly 26, 1999; Revised Manuscript Received November 8, 1999
}

\begin{abstract}
Recent experiments on the pullout of single polymer chains have revealed a complex behavior of the force fluctuations. This paper analyzes the pullout process theoretically and numerically and shows that these fluctuations can be made to shed light on disentanglement dynamics. To facilitate the analysis, I first derive the probability density function of the threshold force needed to disentangle one entanglement point. This function is found to be dominated by large fluctuations, which bears directly on the observed statistics. The average and variance of the force are calculated, and a numerical investigation of the dynamics is carried out to check the results. Finally, applications to deformations in several macroscopic systems are discussed.
\end{abstract}

\section{Introduction}

Recent measurements of force fluctuations during pullout of single polymer chains have brought to light some fundamental issues concerning their dynamics. ${ }^{1}$ In a typical such experiment, an AFM cantilever beam is brought close to a polymeric surface and the tip bonds chemically to a chain. Bonding to only one chain is achieved by a careful choice of the system conditions and the tip material. The cantilever beam then retreats, and the force on it is measured. Traditionally, chain dynamics are presumed to consist of sliding along an effective tubelike channel formed by entanglements with other chains. ${ }^{2}$ This picture, combined with equilibriumbased arguments, makes it possible to derive many results and, in particular, the typical friction force that the chain needs to overcome in the process. Pulling a single chain, however, invol ves nonequilibrium dynamics and therefore cannot be understood within this traditional picture. Moreover, the assumption that the disentanglement force has a typical value dominates many studies, ${ }^{3}$ yet this assumption becomes increasingly questionable as the temperature is reduced toward the glass transition and cannot be applied safely to single chains. Thus, the distribution of disentanglement forces is a crucial issue for the single chain pulling (SCP) dynamics, as well as for more general issues. Pullout of polymer chains at temperatures around, and below, the glass transition is at the heart of many macroscopic processes. Examples are failures of polymer/polymer and polymer/matrix interfaces, as occurring in rubber and fiber enhanced polymers, welded polymers, and debonding of adhesives. Thus, understanding the SCP dynamics is an essential first step to understanding these issues.

Here, I model this process and derive the distribution of the force fluctuation measured under constant pulling rate. Although SCP by an AFM cantilever beam does not correspond exactly to this condition, it has the advantage of both being convenient to analyze and a reasonable approximation. The base of the cantilever beam is moved at a constant speed, and the deflection of the beam due to the tugging at the tip is used to measure the force. It follows that the tip that pulls the chain does not move at a constant speed nor is it pulling

\footnotetext{
*E-mail: rbbll@phy.cam.ac.uk.
}

at a constant force. Thus, the boundary conditions in the actual experiment consist of neither constant strain nor constant stress rates. Nevertheless, because the stiffness of most beams is on the order of $1-10 \mathrm{Nm}^{-1}$ and the forces involved in the pullout process are at most on the order of nanonewtons, then a constant strain rate is a plausible approximation. A numerical study that takes into account the true boundary conditions within the present model is under construction. For clarity, the model is first described and analyzed at zero temperature. An essential ingredient in the present theory is the distribution of the threshold forces of disentangl ement, and I therefore derive it here using a simple microscopic argument. The probability density function (PDF) of the threshold forces is found to have no local maximum but rather to decay algebraically. This result has significant implications on models that use a typical value or a Gaussian distribution for the disentanglement forces, and these implications are briefly discussed here. A more thorough discussion with suggestions for experimental testing of this finding will be given elsewhere. ${ }^{4} \mathrm{~A}$ numerical simulation of the process is constructed to test the calculations, and the agreement is excellent. Next, the model is extended to include effects of temperature. It is argued that there are two main effects: an appearance of an entropic force in the uncoiling stage and a modification of the probability of disentanglement due to thermal fluctuations. These two effects are discussed and analyzed. To conclude, I discuss the application of the result to the following: (a) failure stress and the growth of a plastically deformed zone in polymer glasses; (b) separation of welded polymers; and (c) failure along polymer/matrix interfaces.

\section{The Model}

Here, disentanglement is assumed to be a local process. This means that during disentanglement the force is transmitted through one (the primary) chain from the pulling point to an entangled point. The force builds up locally at that point until it is disentangled. Once that happens, further pulling of the primary chain continues. Although outside the assumptions of the present model, it should be mentioned that a nonlocal disentanglement process may also occur, whereupon increasing the force on the entangled point leads to 
pulling of the anchor chains before the point is disentangled. The force is then distributed over several entanglement points that are not necessarily on the same chain. These points are connected through a tree that includes the primary and secondary strained chains. Two scenarios are then possible:

(i) The force on an entangl ement point along the tree exceeds the local threshold (see calculation of the threshold forces distribution below). Disentanglement of that point occurs, at the conclusion of which there is a sharp increase of the force on the nearby entangled points. This, in turn, may lead to a disentanglement of another point and so on, resulting in an avalanche. The statistics of avalanche sizes would be interesting to study and may even exhibit a self-organized distribution under suitable conditions.

(ii) Due to the force redistribution, each entangled point carries a stress that is below its threshold. However, if the force is transmitted to several entanglement points via one of the chains in the tree, this force may reach scission level before any of the individual entanglement points disentangles and the chain could snap.

Thus, a nonlocal disentanglement process leads to rich dynamics, but these are outside the scope of this work and are left to a later study. It should be commented, however, that the analysis presented here forms a basis for addressing issues concerned with nonlocal disentanglement.

The system under consideration is an ensemble of entangled polymer chains, where a chain typically occupies a free volume of linear size R. In equilibrium, this would be the radius of gyration, $R_{g}$. From this ensemble, a single chain is being pulled out. It is useful to first view the dynamics of the pulling process mechanistically and introduce effects of temperature later on. The chain is imagined to be entangled at particular entanglement points (EPs), indexed by $n=$ $1,2, \ldots, N_{e p}$, with a chain length of $I_{n}$ forming the nth segment between the $(n-1)$ th and the nth points, and this length is measured in units of Kuhn's length a. The pulling point can be labeled as $n=0$. Between any two such points, the polymer chain is presumed to be coiled with a Gaussian distribution. Generally, pulling at a point anywhere along the chain may result in simultaneous pulling on both sides of the point with disentanglements occurring along both legs where a leg is the part of the chain extending from its end to the pulling point. For clarity, it is assumed here that disentanglements occur only al ong one leg of the chain as it is pulled. The statistics of pulling two legs are slightly more involved but still manageable by building on the analysis presented below. The process begins with a segment of the chain being pulled out at a constant speed, v. The first coiled segment starts to uncoil, which occurs without resistance (a resistive force appears with increasing temperature when entropic effects come into play). After a time interval of

$$
\mathrm{t}_{1}=\mathrm{I}_{1} / \mathrm{v}
$$

the first segment has been completely uncoiled. Note that the first segment may be uncharacteristically short, but this detail should be insignificant when the number of EPs is not anomalously small. Now, a force starts to build up on the first EP. The force is transmitted through the stretched part of the chain whose stiffness is $k_{s} / l_{1}$, where $k_{s}$ is Young's modulus. During this stage, the force on node 1 increases continuously with time at a rate

$$
\dot{\mathrm{F}}=\mathrm{k}_{\mathrm{s}} \mathrm{v} / \mathrm{I}_{1}
$$

When the force reaches a threshold value, $f_{1}$, the node disentangles and the second segment starts to uncoil. The disentanglement time is defined as the time that it takes to reach $f_{1}$ and is given by

$$
t_{d, 1}=\frac{f_{1} l_{1}}{k_{s} v}
$$

During this time, the chain is not pulled out. Rather, its already uncoiled part stretches elastically to an additional length of $\left(f_{1} I_{1}\right) / k_{s}$, which is small due to the high value of $\mathrm{k}_{\mathrm{s}}$. Immediately after the node disentangles, the chain relaxes back. After another uncoiling period, the second segment becomes fully stretched, the next EP feels an increasing stress, and it disentangles when the force reaches $f_{2}$. The process repeats itself until the entire chain is stretched out. The time between the disentanglements of two successive points, $n-1$ and $\mathrm{n}$ is

$$
T_{n}=t_{u, n}+t_{d, n}=\frac{I_{n}}{v}+\frac{f_{n} \sum_{j=1}^{n(t)} I_{j}}{k_{s} v}
$$

where $t_{u, n}$ is the uncoiling time of the $n$th segment, $t_{d, n}$ is the disentangl ement time of the nth $E P, \sum_{j=1}^{n(t)} l_{j}$ is the length of the chain pulled out after $n(t)$ such events. Since the force on the nth EP increases linearly with time between 0 and $f_{n}$ then the force inside this interval is $f_{n} \delta t_{n} / t_{d, n}$, where $\delta t_{n}$ is the time from the beginning of disentanglement. It follows that the force on the chain at each moment is

$\mathrm{F}(\mathrm{t})=$

$$
\sum_{n=1}^{N(t)}\left[H\left(t-\Theta_{n-1}\right)-H\left(t-\Theta_{n-2}-t_{u, n}\right)\right] \frac{\delta t_{n}}{t_{d, n}} f_{n}
$$

where $\Theta_{\mathrm{n}}=\sum_{\mathrm{k}=1}^{\mathrm{n}}\left(\mathrm{t}_{\mathrm{u}, \mathrm{k}}+\mathrm{t}_{\mathrm{d}, \mathrm{k}}\right)$ and $\mathrm{H}(\mathrm{x})$ is the Heavyside step function that is unity for $\mathrm{x}>1$ and vanishes for $\mathrm{x}$ $<1$. From eq 4, the distribution of the time intervals, $t_{u, n}=I_{n} / v$, depends only on the distribution of $I_{n}$. The distribution of $t_{d, n}$ depends both on the distribution of forces, $f_{n}$, and on the distribution of the length, $\sum_{j=1}^{n} l_{j}$. Because the distribution of $I_{n}$ is narrow, its PDF, $P_{1}\left(I_{n}\right)$, can be safely assumed to be Gaussian. ${ }^{5}$

$$
P_{1}\left(I_{n}\right)=C_{1} \exp \left[-\frac{\left(I_{n}-I_{0}\right)^{2}}{2 \sigma_{1}^{2}}\right] \quad I_{\text {min }}<I_{n}<I_{\max }
$$

where $0<I_{\min } \ll I_{0} \ll I_{\max }$ and $\sigma_{\mid}$is the width of the Gaussian distribution. The normalizing factor $C_{1}$ is, to an exponentially good accuracy, $1 /(2 \pi)^{1 / 2} \sigma_{\mid}$.

\section{The PDF of the Disentanglement Threshold Forces}

An entanglement occurs at a point where the chain is wrapped around an anchor chain (or chains) over an angle that lies between 0 and $2 \pi$, as sketched in Figure $1 .{ }^{6}$

The black bead in Figure 1 represents the projection of the anchor, which is oriented locally normal to the 


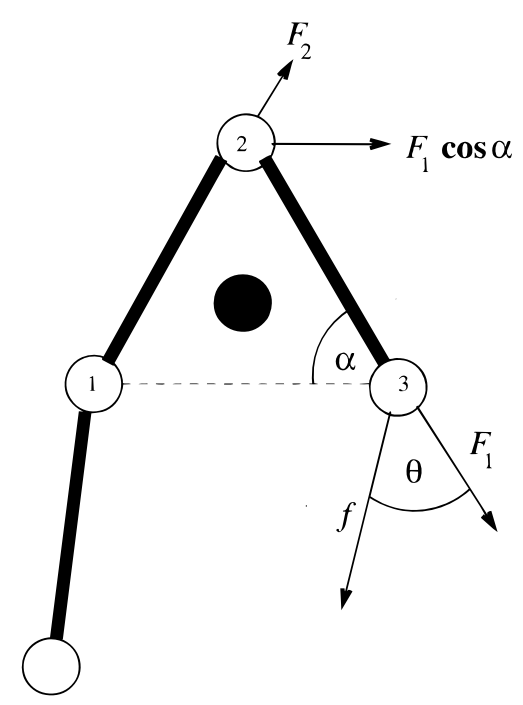

Figure 1. Disentanglement configuration. The black circle is an anchor chain perpendicular to the page plane around which the disentangling chain moves.

wrapping (page) plane. The orientation of the anchor effectively reduces the dimensionality of the kinetics, and the force that pulls the white-beaded chain can be regarded as lying in the page plane. Even if this is not initially the case (meaning that the force has a component along the anchor), or when the anchor is initially mobile, the pulling force will cause a local adjustment of the chains until the anchor chain cannot move any further. Thus, the anchor is immobile throughout the process of interest. Disentanglement occurs when beads 1 and 2 move one position cl ockwise around the anchor . If the activation energy needed to move one segment of the chain (say, from position 1 to 2 in Figure 1 ) is defined as $E_{b}$, there is a barrier of $\mathrm{mE}_{b}$ to climb in order to initiate a displacement of $m$ segments of the chain in contact with the anchor. The anchor may consist of one chain, in which case $m$ is expected to be 2 , or it may consist of a bunch of chains, in which case $m$ may be larger (but the barrier $\mathrm{E}_{\mathrm{b}}$ may be lower). For clarity, it is assumed here that $m=2$. Consider now a force $f$ tugging at bead 3 . The active component pulling on the entanglement point is

$$
\mathrm{F}_{1}=\mathrm{f} \cos \theta
$$

In an isotropic medium, the angle $\theta$ is uniformly distributed, and so, $F_{1}$ can take, in principle, any value between $f$ and 0 . On gradual increase of $f$, a threshold is reached, and the chain starts moving. The force $F_{2}=$ $F_{1}$ pulls on the segment between 2 and 1 , and the angle $\alpha$ (presumed fixed) is determined by the details of the molecular potentials. For simplicity, it is assumed here that once a chain starts slipping it will continue to do so and the EP disappears. It is possible to modify this picture to a more elaborate mechanism of disentanglement, but this model suffices to capture the essential features. The slipping criterion is that slipping occurs when the work needed to move the chain by $\mathrm{a}, 2 \mathrm{aF}_{1}$, is equal to the activation energy $2 \mathrm{E}_{\mathrm{b}}$, or $\mathrm{F}_{1}=\mathrm{E}_{\mathrm{b}} / \mathrm{a}$. $\mathrm{E}_{\mathrm{b}} / \mathrm{K}_{\mathrm{B}}$ ( $\mathrm{k}_{\mathrm{B}}$ being Boltzmann constant) is expected to be somewhat above the glass-transition temperature and only weakly (or not at all) dependent on molecular weight. For polystyrene, it is estimated here roughly as $400 \mathrm{~K}$. The value of a can be taken as $5 \AA$. This puts $F_{1}$ approximately at a value of $11 \mathrm{pN}$. We now need to find the probability density of $f$ needed to provide the component $F_{1}$. The distribution of the value of $f$ stems from the randomness of the angle $\theta$ that the global pullout direction makes with the local direction of $F_{1}$. In an isotropic medium $\theta$, is uniformly distributed between 0 and some $\theta_{\max }<\pi / 2$

$$
\mathrm{P}_{\theta}(\theta)=1 / \theta_{\max }
$$

The reason for not considering $\theta<0$ is that a force in this direction leads to uncoiling, rather than a sliding motion around the anchor chain and therefore does not entail disentanglement. The reason for disregarding values of $\theta$ that are too cl ose to $\pi / 2$ is that in this regime the force is almost normal to $F_{1}$ and will only serve to adjust the position of the anchored chain. If the anchor chain cannot be adjusted further, then the force needed to provide a component of size $F_{1}$ is too large and scission will occur before sliding. In fact, we can designate this as a condition for $\theta_{\max }$

$$
\cos \theta_{\max }=\mathrm{F}_{1} / \mathrm{F}_{\text {scission }} \equiv \epsilon
$$

where $F_{\text {scission }}$ is the force required for the scission of one chain. Because $\theta_{\max }$ is close to $\pi / 2$, then $\epsilon \ll 1$. From eq 7 and the distribution of $\theta$, we can now deduce the PDF of $f$.

$$
\begin{aligned}
P_{f}(f)=\frac{1}{|d f / d \theta|} P_{\theta}(\theta)= & \frac{1}{\theta_{\text {max }} f \sqrt{\left(f / f_{\text {min }}\right)^{2}-1}} \mathrm{f}_{\text {min }} f \leq f_{\text {max }}
\end{aligned}
$$

where $f_{\min }=F_{1}$ and $f_{\max }=f_{\min } / \cos \theta_{\max }$. The significant feature to note is the non-Gaussian form of this PDF for both large and small $f$. For $f=f_{\min }+\delta f$, where $\delta f \ll$ $f_{\text {min }}$, the PDF diverges as $P_{f}(f) \sim 1 /(\delta f)^{1 / 2}$. For $f \rightarrow f_{\text {max }}$, the PDF decays as $P_{\mathrm{f}}(\mathrm{f}) \sim 1 / \mathrm{f} 2$.

The clearest manifestation of the non-Gaussian behavior of the force distribution comes from the cal culation of the moments,

$$
\left\langle f{ }^{k}\right\rangle=\int_{f_{\min }}^{f_{\max }}{ }^{k} P_{f}(f) d f
$$

The first few moments are

$$
\begin{aligned}
& \langle\mathrm{f}\rangle=\frac{\mathrm{f}_{\min }}{\theta_{\max }} \ln \frac{1+\sin \theta_{\max }}{1-\sin \theta_{\max }} \quad\left\langle\mathrm{f}^{2}\right\rangle=\frac{\mathrm{f}_{\min }{ }^{2} \tan \left(\theta_{\max }\right)}{\theta_{\max }} \\
& \left\langle\mathrm{f}^{3}\right\rangle=\frac{\mathrm{f}_{\text {min }}{ }^{3}}{4 \theta_{\max }}\left[\frac{2 \sin \left(\theta_{\max }\right)}{\cos ^{2}\left(\theta_{\max }\right)}+\ln \frac{1+\sin \theta_{\max }}{1-\sin \theta_{\max }}\right] \\
& \left\langle f^{4}\right\rangle=\frac{f_{\min }^{4}}{3 \theta_{\max }}\left[\tan ^{3}\left(\theta_{\max }\right)+3 \tan \left(\theta_{\max }\right)\right]
\end{aligned}
$$

All the moments are dominated by the upper limit defined in eq 8 ,

$$
\langle\mathrm{f}\rangle \sim \ln (1 / \epsilon) \quad\left\langle\mathrm{f}^{\mathrm{k}}\right\rangle \sim 1 / \epsilon^{\mathrm{k}-1}
$$




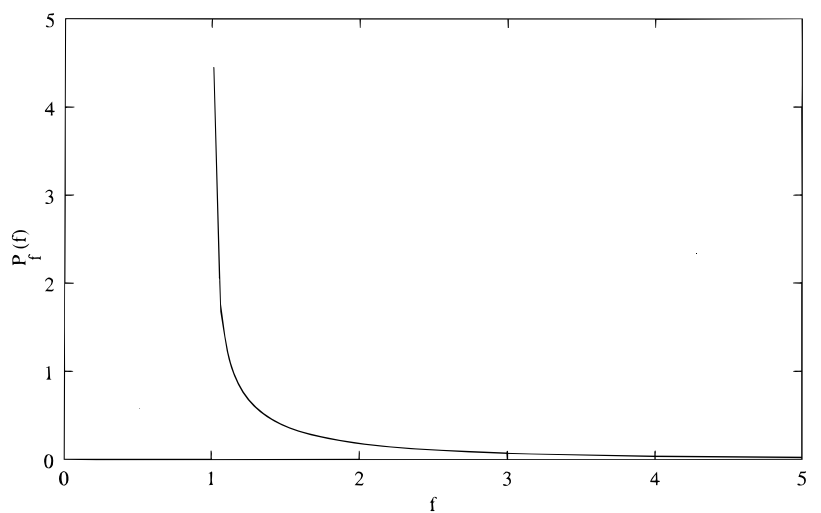

Figure 2. PDF of the normalized force $P_{\phi}(\phi)$.

The variance (the second cumulant) of the PDF is

$$
\begin{array}{r}
\left\langle\delta^{2} \mathrm{f}\right\rangle=\left\langle\mathrm{f}^{2}\right\rangle-\langle\mathrm{f}\rangle^{2}=\left(\frac{\mathrm{f}_{\min }}{2 \theta_{\max }}\right)^{2}\left[4 \theta_{\max } \tan \left(\theta_{\max }\right)-\right. \\
\left.\ln ^{2} \frac{1+\sin \theta_{\max }}{1-\sin \theta_{\max }}\right] \sim \frac{1}{\epsilon}
\end{array}
$$

which is also sensitive to the large fluctuations. The conclusion is then that the PDF is dominated by large fluctuations and cannot be approximated by a typical value of $f$. This casts some doubt on traditional models that use the tube model with a typical friction force for describing disentanglement in too low a range of temperatures.

To plot the PDF of $f$, it is convenient to scale $f$ by defining the variable $\phi \equiv \mathrm{f} / \mathrm{f}_{\min }$. The PDF of $\phi$ has a universal form

$$
\mathrm{P}_{\phi}(\phi) \mathrm{d} \phi=\frac{\mathrm{d} \phi}{\theta_{\max } \phi \sqrt{\phi^{2}-1}} \quad 1 \leq \phi \leq 1 / \cos \left(\theta_{\max }\right)
$$

that is plotted for $\theta_{\max }=\pi / 2-0.01$ in Figure 2 .

\section{Analysis}

With the distributions of the threshold forces and the coiled lengths explicitly known, it is possible now to calculate the statistics of the dynamical process. The PDF of the uncoiling time intervals is straightforwardly related to that of the uncoiled lengths,

$$
\mathrm{P}_{\mathrm{u}}\left(\mathrm{t}_{\mathrm{u}}\right)=\frac{\mathrm{v}}{\sqrt{2 \pi} \sigma_{1}} \exp \left[-\frac{\left(\mathrm{t}_{\mathrm{u}}-\mathrm{t}_{0}\right)^{2} \mathrm{v}^{2}}{2 \sigma_{1}^{2}}\right]
$$

The PDF of the time interval between two successive disentanglements, $T_{n}=t_{u, n}+t_{d, n}$, is slightly more involved. Formally, it can be presented as

$$
\begin{aligned}
P_{T}\left(T_{n}\right)=\int_{f_{\min }}^{f_{\max }} P_{f}\left(f_{n}\right)\left[\prod_{j=1}^{n} \int P_{1}\left(I_{j}\right) d l_{j}\right] \delta\left[T_{n}-\right. \\
\left.\left(\frac{\left(1+f_{n} / k_{s}\right)}{v} I_{n}+\frac{f_{n}}{v k_{s}} \sum_{j=1}^{n-1} I_{j}\right)\right] d f_{n}
\end{aligned}
$$

For brevity, I drop the subscript $\mathrm{n}$ from $\mathrm{T}_{\mathrm{n}}$ except where it may lead to confusion. Expressing the $\delta$-function as a Fourier integral and integrating over the PDFs of $I_{j}$ gives

$$
P_{T}(T)=\int_{f_{\text {min }}}^{f_{\text {max }}} P_{f}\left(f_{n}\right) \frac{1}{\sqrt{2 \pi} b_{n}} \exp \left[-\frac{\left(T-a_{n}\right)^{2}}{2 b_{n}^{2}}\right] d f_{n}
$$

where $a_{n}=\left(1+\mathrm{nf}_{\mathrm{n}} / \mathrm{k}_{\mathrm{s}}\right) \mathrm{l}_{0} / \mathrm{v}$ and $\mathrm{b}_{\mathrm{n}}=\left(\sigma_{\mathrm{l}} / \mathrm{v}\right)\left[\mathrm{n}\left(\mathrm{f}_{\mathrm{n}} / \mathrm{k}_{\mathrm{s}}\right)^{2}+2 \mathrm{f}_{\mathrm{n}} /\right.$ $\left.\mathrm{k}_{\mathrm{s}}+1\right]^{1 / 2}$. Using this expression, the moments of $\mathrm{T}$ are

$$
\left\langle T^{k}\right\rangle=\int T^{k} P_{T}(T) d T=\int_{f_{\min }}^{f_{\max }} d f_{n} P_{f}\left(f_{n}\right)\left[T^{k}\right]_{g}
$$

where

$$
\left[T^{k}\right]_{g}=T^{k} \frac{1}{\sqrt{2 \pi} b_{n}} \exp \left[-\frac{\left(T-a_{n}\right)^{2}}{2 b_{n}^{2}}\right] d T
$$

are averages over the Gaussian kernel in eq 15. For example,

$$
\begin{gathered}
\langle T\rangle=\int_{f_{\text {min }}}^{f_{\max }} P_{f}(f) a_{n}(f) d f=\left(1+\frac{n}{k_{s}}\langle f\rangle\right) \frac{I_{0}}{v} \\
\left\langle T^{2}\right\rangle=\int_{f_{\text {min }}}^{f_{\text {max }}} P_{f}(f)\left(b_{n}{ }^{2}+a_{n}{ }^{2}\right) d f= \\
\langle T\rangle^{2}+\left(\frac{\sigma_{I}}{v}\right)^{2}\left[\frac{n}{k_{s}{ }^{2}}\left\langle f^{2}\right\rangle+\frac{2}{k_{s}}\langle f\rangle+1\right]
\end{gathered}
$$

where $\langle f\rangle$ and $\left\langle f^{2}\right\rangle$ are the first and second moments of the force distribution $P_{f}(f)$. Because the moments $\langle f k\rangle$ $\sim 1 / \epsilon^{k-1}$ are dominated by large fluctuations, then so are the moments of $T_{n}$. Moreover, because $1 / \epsilon=F_{\text {scission }} /$ $F_{1}$, it follows that

$$
\left\langle T^{k}\right\rangle \sim\left\langle\mathrm{f}^{\mathrm{k}}\right\rangle \sim\left(\frac{\mathrm{F}_{\text {scission }}}{\mathrm{F}_{1}}\right)^{\mathrm{k}-1}
$$

which gives a rather unexpected dependence of these moments on the chain scission threshold.

The time that it takes the chain to go through $\mathrm{N}$ uncoiling-disentanglement events is $\Theta_{N}=\sum_{n=1}^{N} T_{n}$, whose PDF can be readily found using the above relations:

$P_{\Theta}(\Theta)=\int_{f_{\min }}^{f_{\max }} \exp \left[-\frac{\left(\Theta-\Theta_{0}\right)^{2}}{2 \Gamma^{2}}\right]\left[\prod_{n=1}^{N} \int P_{f}\left(f_{n}\right) d f_{n}\right]$

In this expression, $\Theta_{0}=\sum_{n=1}^{N} a_{n}$ and $\Gamma^{2}=\sum_{n=1}^{N} b_{n}{ }^{2}$, and both can be computed straightforwardly from the above expressions for $a_{n}$ and $b_{n}$. The moments of $\Theta_{N}$ are best evaluated from those of $T_{n}$; for example, the mean time to free $\mathrm{N}$ segments is

$$
\left\langle\Theta_{N}\right\rangle=\sum_{n=1}^{N}\left\langle T_{n}\right\rangle=N \frac{I_{0}}{v}\left(1+\frac{N-1}{2} \frac{\langle f\rangle}{k_{s}}\right)
$$

All of the above analysis holds as long as the total freed length is smaller than the entire length of the pulled leg. If the pulling point happens to be at a chain's end, then only one side of the chain is pulled and $\mathrm{N}<$ $M_{d} / M_{e}$, where $M_{c}$ and $M_{e}$ are, respectively, the chain and entanglement molecular weights. Since the pulling point is more likely to occur at a point somewhere along the chain, then disentanglement processes can take place along either one or both legs. In the former case, $\mathrm{N}$ is bound by the number of EPs between the pulling point 
and the end of the chain al ong the pulled leg. The latter case requires some extension of the present statistical analysis and will be addressed elsewhere. Once the chain is fully stretched, further pullout is disentanglement-free and takes place by sliding against friction with other chains. Crossing over to this mechanism during an experiment results in a distinct change of the force statistics, which is bound to be observable. Let us calculate the expected average force needed to mai ntain the constant rate, v. During the uncoiling period, the chain offers very little resistance compared to the disentanglement periods, which therefore will be neglected here altogether. During the nth disentanglement period, the force increases gradually from 0 to $f_{n}$. The average force is obtained by time averaging of $\mathrm{F}(\mathrm{t})$ (eq 5) over a time $\Theta_{N}$ :

$$
\bar{F}=\frac{1}{\Theta_{N}} \int_{0}^{\Theta_{N}} F(t) d t
$$

This integral is evaluated in Appendix A and, to secondorder in $\langle f\rangle / k_{s}$, it gives

$$
\begin{aligned}
& \bar{F}=\frac{(N-1)}{4 k_{s}}\left\langle f^{2}\right\rangle- \frac{1}{4 k_{s}^{2}}\left[\frac{(N-1)^{2}}{2}\left\langle f^{2}\right\rangle\langle f\rangle+\right. \\
&\left.\frac{(N+1)(2 N+1)}{3 N}\left(\left\langle f^{3}\right\rangle-\left\langle f^{2}\right\rangle\langle f\rangle\right)\right]
\end{aligned}
$$

The reason for expanding in this variable is that $\langle\mathrm{f}\rangle / \mathrm{k}_{\mathrm{s}}$ has to be small or else scission occurs. Equation 22 presents us with a surprise: to lowest order, the average force is proportional to the second moment of the PDF of the threshold forces rather than to the mean. Because that PDF is dominated by large fluctuations

$$
\left\langle\mathrm{f}^{2}\right\rangle=\frac{\mathrm{f}_{\min }^{2}}{\theta_{\max }} \tan \theta_{\max } \approx \frac{2 \mathrm{f}_{\min }^{2}}{\pi \epsilon}
$$

then this is immediately reflected in the average pulling force. Because $\epsilon$ is small, $\bar{F}$ is large, which is good news for AFM measurements. It should be noted that although $\epsilon$ is small, the product $k_{s} \epsilon$ is still larger than $f_{\min }$, again, due to the condition that the forces on the chain are bel ow the scission threshold. One can similarly calculate the average of the second moment

$$
\begin{aligned}
\overline{\delta^{2} \mathrm{~F}}=\overline{\mathrm{F}^{2}}-\overline{\mathrm{F}}^{2}= & \\
& \frac{1}{\Theta_{\mathrm{N}}} \int_{0}^{\Theta_{\mathrm{N}}}\left[\mathrm{F}^{2}(\mathrm{t})-\frac{1}{\Theta_{\mathrm{N}}} \int_{0}^{\Theta_{\mathrm{N}}} \mathrm{F}\left(\mathrm{t}^{\prime}\right) \mathrm{dt}\right] \mathrm{dt}
\end{aligned}
$$

but presenting this expression in a simpler analytical form is less straightforward and is left for a later report.

Let us calculate next the total work needed to pull out a chain at zero temperature, $\bar{W}_{c}$. First, consider the work needed to disentangle the nth EP,

$$
W_{n}=\frac{1}{2} \frac{f_{n}^{2}}{\left(k_{s} / \sum_{j=1}^{n} l_{j}\right)}
$$

where the denominator represents the spring constant at the nth stage. Summing over N EPs and averaging over the PDFs of $f_{n}$ and $l_{j}$, the total work is found to be

$$
\begin{aligned}
\mathrm{W}_{\mathrm{c}}=\left\langle\sum_{\mathrm{n}=1}^{\mathrm{N}} \mathrm{W}_{\mathrm{n}}\right\rangle=\frac{\left\langle\mathrm{f}^{2}\right\rangle \mathrm{I}_{0}}{2 \mathrm{k}_{\mathrm{s}}} \sum_{\mathrm{n}=1}^{\mathrm{N}} \mathrm{n}= \\
\frac{\mathrm{N}(\mathrm{N}+1) \mathrm{I}_{0} \mathrm{f}_{\min }{ }^{2} \tan \left(\theta_{\max }\right)}{4 \mathrm{k}_{\mathrm{s}}} \approx \frac{\mathrm{N}^{2} \mathrm{f}_{\text {min }}{ }^{2} \mathrm{I}_{0}}{2 \pi \epsilon \mathrm{k}_{\mathrm{s}}}
\end{aligned}
$$

Note the dependence on the second moment of the PDF $P_{f}(f)$ that is very large due to the significant contribution of large fluctuations. It follows that the work is much larger than what one would expect from a typical value of $f$.

\section{Numerical Analysis of the Dynamics}

To check the above analysis, a numerical code has been constructed that simulates the dynamics of a chain as it is being pulled out from the bulk at a constant rate. The chain is modeled as a one-dimensional line, and a set of entanglement points is determined along the chain by choosing the length of the segments from the normal distribution given in eq 6 . Three values of $I_{0}$ have been studied: $I_{0}=100,500$, and 1000 . For each distribution, two widths were simulated: $\sigma_{1}=0.11_{0}$ and $0.3 \mathrm{l}_{0}$. The chain is assumed to be sufficiently long that it does not become fully stretched during the simulation time. For each EP, a threshold disentanglement force is chosen randomly from the PDF $P_{f}(f)$, where $f_{m i n}$, is normalized to 1 and $\theta_{\max }=\pi / 2-0.01$ (corresponding to $\mathrm{f}_{\max } \approx 100 \mathrm{f}_{\min }$ ). The pullout rate has been normalized to $v=1$. Given a particular chain with a choice of segments and threshold forces distributions, it is determined at each time step whether the chain is in an uncoiling or disentangling state. The force is then calculated, and a force time series is constructed. A typical such plot is shown in Figure 3. The plot shows large fluctuations of the pulling force, as expected from the PDF (see eq 9). The effect of changing the width of $P_{I}(1)$ has been found to be negligible for values of $N$ already of order 10 . The mean force, $\bar{F}$, has been computed numerically for 10000 chains with $I_{0}=1000$ and after $\mathrm{N}=500$ disentanglements. The resulting distribution is plotted in Figure 4. Equation 22 predicts for these parameters that the maximum will be at approximately

$$
\overline{\mathrm{F}} \approx \frac{\mathrm{N}\left\langle\mathrm{f}^{2}\right\rangle}{4 \mathrm{k}_{\mathrm{s}}} \approx 0.2
$$

where the correction term of order $1 / k_{s}^{2}$ has been neglected. The plot agrees very nicely with the theoretical prediction.

Next, I compute the mean work, $\overline{\mathrm{W}}_{\mathrm{c}}$, needed to pull one chain through 500 disentangl ements, with the same chain parameters. From eq 25 , the average work after 500 disentanglement events is approximately $\overline{\mathrm{W}}_{\mathrm{c}} \approx$ $82 \mathrm{I}_{0}$. The distribution of $\overline{\mathrm{W}}_{\mathrm{d}} \mathrm{l}_{0}$ over 10000 chains is shown in Figure 5 . The peak occurs at about $100 \mathrm{I}_{0}$, in reasonable agreement with this estimate.

\section{Effects of Temperature}

Temperature introduces two effects: First, the uncoiling process is no longer force-free due to the entropic contribution to the free energy. Second, the probability of disentanglement changes due to the assistance of thermal fluctuations in getting the beads of the pulled 


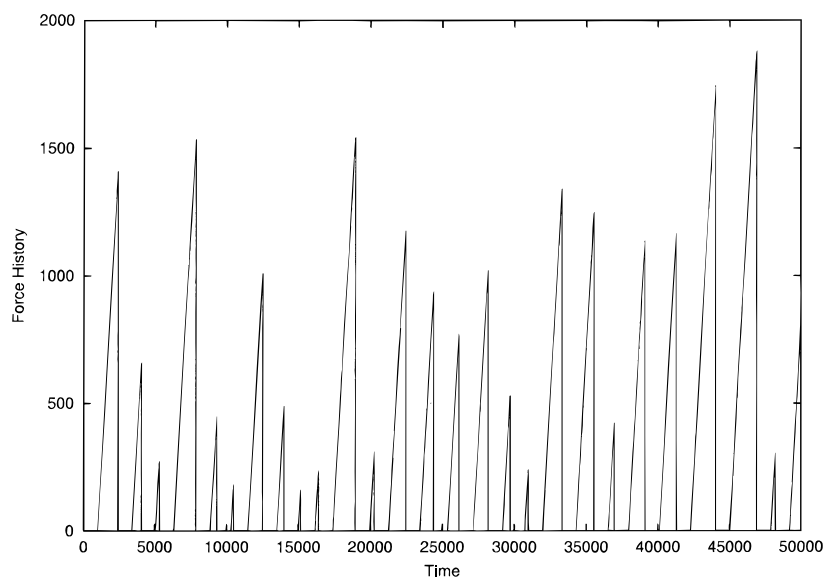

Figure 3. Typical behavior of $F(t)$ for an arbitrary chain. Note the large fluctuations indicating that a typical force is not a good descriptor.

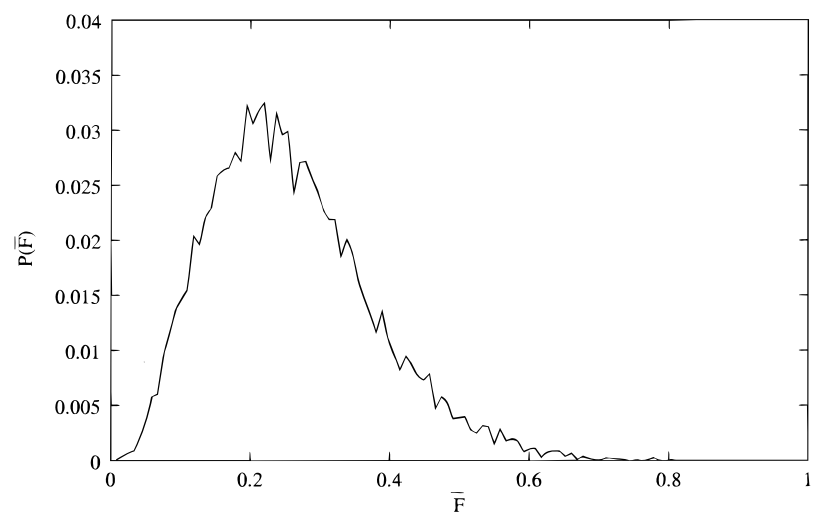

Figure 4. Distribution of $\overline{F(t)}$ after 500 disentanglements over 10000 pullout processes. The mean length between disentanglements is $I_{0}=1000$. The distribution peaks at $\bar{F}=$ 0.2 , in good agreement with eq 22.

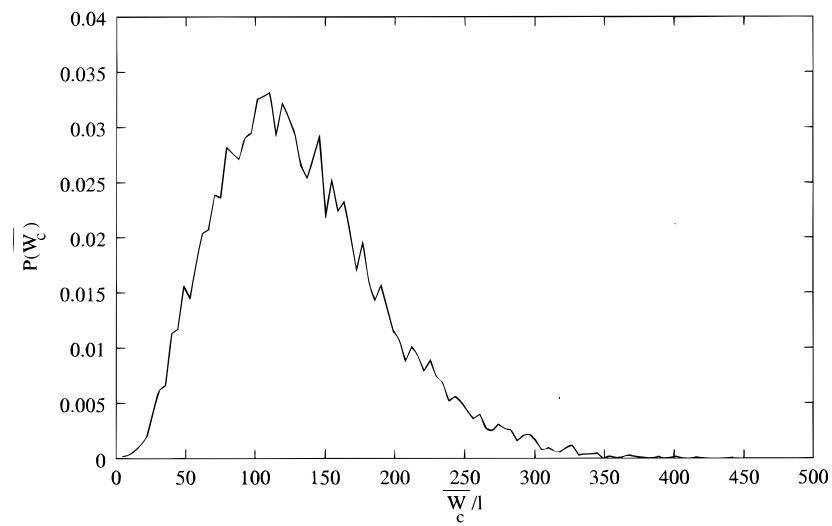

Figure 5. Distribution of $\overline{\mathrm{W}_{d}} \mathrm{l}_{0}$ with the same parameters as in Figure 4. The peak is at 100 in reasonable agreement with eq 25.

chain over the potential barrier. Other effects are neglected here, e.g., the temperature dependence of the distribution of chain lengths between EPS.

For the uncoiling process, it is a textbook exercise ${ }^{7}$ to show that stretching a freely jointed chain of length $L$ $=N_{e} a$, whose end-to-end distance is $R$, leads to a reduction in phase space that increases the free energy. The force needed for such a stretch is the derivative of the free energy with respect to $R$ and has been found to be $^{7}$

$$
f_{u}=\frac{k_{B} T}{a} L^{-1}\left(\frac{R}{L}\right)
$$

where $k_{B}$ is Boltzmann's constant and $L^{-1}(x)$ is the inverse of the Langevin function

$$
L(x)=\operatorname{coth} x-1 / x
$$

By assuming a speed that is sufficiently slow to allow the nth segment to relax yet not so sl ow as to allow the entire pulled out length to equilibrate, the end-to-end distance is the vector between the $(n-1)$ th and nth $E P s, R \approx\left(I_{n} a\right)^{1 / 2}$. The uncoiling of the nth segment can then be envisaged as increasing $R$ with time and to a good accuracy

$$
\frac{f_{u} a}{k_{B} T}=L^{-1}\left(\frac{\sqrt{I_{n} a}+v \delta t}{I_{n}}\right)
$$

where $\delta \mathrm{t}$ is the time from the start of the uncoiling process. This expression will hold until $\mathrm{R}$ becomes about $1 / 3$ of $I_{n}$. Because of the appearance of a finite force during the uncoiling intervals, the behavior of the force as a function of time is smoother than the zero temperature saw-tooth pattern.

Next, let us consider temperature effects on the disentanglement probability. Recall that at zero temperature the chain could slide over the anchor only when the component of the force $F_{1}$ in Figure 1 exceeded the threshold $F_{1}=E_{b} / a$. At finite temperatures, thermally assisted hopping over the energy barrier leads to a finite probability of disentanglement occurring even when $F_{1}$ is bel ow this threshold. Given a force $F_{1}$, the probability of jumping over the barrier is

$$
p=\exp \left[-m\left(E_{b}-a F_{1}\right) / k_{B} T\right]
$$

when $\mathrm{aF}_{1}<\mathrm{E}_{\mathrm{b}}$ and $\mathrm{p}=1$ otherwise. Here, $\mathrm{maF}_{1}$ is the work done by the force $F_{1}$ when moving the chain by one unit a. Note that if the anchor consists of more than one chain then the barrier height to overcome changes somewhat and, more interestingly, may also be distributed statistically. The probability of disentanglement is found by integrating $\mathrm{pP}_{\theta}(\theta)$ over the range $0<\theta<$ $\theta_{\max }$, taking careful consideration of the fact that $p$ is nonanalytic and saturates to unity at a value of $\theta$ that depends on $\mathrm{f}$. This calculation yields

$$
P_{f}(f, T)=P_{f}(f, 0)+\exp \left[\frac{-m E_{b}}{k_{B} T}\right] S(f, T)
$$

Here,

$$
\begin{aligned}
\mathrm{S}(\mathrm{f}, \mathrm{T})=\int_{0}^{\mathrm{d} \gamma / \mathrm{df}} \exp \left(\text { maf } \cos \theta / \mathrm{k}_{\mathrm{B}} \mathrm{T}\right) \mathrm{d} \theta+ \\
\quad \int_{0}^{\gamma} \frac{\mathrm{ma} \cos \theta}{\mathrm{k}_{\mathrm{B}} \mathrm{T}} \exp \left(\text { maf } \cos \theta / \mathrm{k}_{\mathrm{B}} \mathrm{T}\right) \mathrm{d} \theta
\end{aligned}
$$

and

$$
\gamma=\arccos \left(\mathrm{E}_{\mathrm{b}} / \mathrm{af}\right)
$$

The details of the derivation of this expression will be given elsewhere. ${ }^{8}$ Thus, at finite temperatures, $0<p$ $<1$, and there is a nonvanishing probability of disentanglement essentially at any time as the force increases on the EP. Among other things, this affects the distribu- 
tion of the disentanglement time, $t_{d, n}$, and hence the behavior of the force fluctuations on the chain. The distribution of $t_{d, n}$ is calculated as follows: Since disentanglement may occur in principle at any force, then one should find first the probability of disentanglement when the force is between $f_{n}$ and $f_{n}+d f$, given that the force started to increase from 0 at a given moment. This probability is the product of the probability to not disentangle until the force reaches $f_{n},\left[1-\int_{0}^{f_{n}} P_{f}(f, T)\right.$ df ], and the probability to disentangle exactly when the force reaches $f_{n}$, namely, $P_{f}\left(f_{n}, T\right)$ df. A detailed analysis of this probability and the consequences to the analysis presented here is deferred to a later report.

\section{Discussion}

Understanding the dynamics of single chain pullout and the pertinent force response is important for several reasons. First, it sheds light on the process of disentanglement dynamics in general, which provides a basis for estimating various parameters: the number of entanglements per chain, their distribution along the chains, the distribution of the disentanglement forces and the work needed to pull out a chain, to name a few. Second, it is directly relevant to practical applications, such as failure of polymer/polymer and polymer/matrix interfaces. In such applications, the interfaces are the potentially weak points and failure at these points is a critical issue. The fact that the interface divides two dissimilar materials is not a problem because the above analysis can be carried out separately for each side of the interface. For example, the distributions of $I_{n}$ and $f_{n}$ may have different numerical parameters (although their form is expected to remain the same). Specific applications are polymers enhanced by rubber or fiber inclusions, the interface between a polymer grafted onto an elastomer network, the interface between two wel ded polymers, and so on. The process that governs the failure usually consists of many chains pulled out in parallel, all undergoing disentangl ements as described here. Note that the vel ocity v corresponds in all of these cases to the strain rate on the boundary of the deformation surface and not at the boundary of the entire system. Disentanglement also precedes deformation mechanisms such as crazing and debonding of adhesives. In the former, the process described here is relevant to the prefibrilation stage when individual chains are stretched and drawn into fibrils. Because fibril sizes and spacings are typically comparable to the radius of gyration, only a few chains are drawn into any single fibril and the dynamics described here applies. In the case of debonding of adhesives, the present description applies to the precavitation stage. In view of these important applications, this model is currently being extended to treat chains in parallel. ${ }^{4}$

Acknowledgment. It is a pleasure to thank Prof A. $M$. Donald for many useful and critical discussions that forced me to clarify the ideas presented here. I have also benefitted from discussions with Prof. T. C. B. Mcleish, Prof. R. C. Ball, and Dr. E. Terentjev. Special thanks to $\mathrm{V}$. J . Anderson for critical reading and elimination of embarrassing mistakes.

\section{Appendix A: The Average Pullout Force}

In this appendix, I calculate the time average of the pullout force needed to maintain the constant pullout rate $v$ at zero temperature. Averaging over a time interval $\Theta_{N}$, we have

$$
\begin{array}{r}
\bar{F}=\frac{1}{\Theta_{N}} \int_{0}^{\Theta_{N}} F(t) d t=\frac{\sum_{n=1}^{N} \frac{1}{t_{d, n}} \int_{0}^{t_{d, n}} f_{n} t d t}{\sum_{n=1}^{N} T_{n}}= \\
\frac{\sum_{n=1}^{N} f_{n} t_{d, n}}{2 \sum_{n=1}^{N}\left(t_{u, n}+t_{d, n}\right)}=\frac{\sum_{n=1}^{N}\left(f_{n}^{2} \sum_{n=1}^{N} l_{j}\right)}{2 \sum_{n=1}^{N}\left(k_{s} I_{n}+f_{n} \sum_{n=1}^{N} l_{j}\right)}
\end{array}
$$

Since the random variables $I_{n}$ are Gaussian then, already for $\mathrm{N}$ of order 10 , we can approximate $\mathrm{I}_{\mathrm{n}}$ by $\mathrm{I}_{0}$, which yields in eq A.1

$$
\bar{F} \approx \frac{\sum_{n=1}^{N} \mathrm{nf}_{\mathrm{n}}^{2}}{2 \mathrm{Nk}_{\mathrm{s}}\left(1+\sum_{\mathrm{n}=1}^{\mathrm{N}} \frac{\mathrm{nf}_{\mathrm{n}}}{\mathrm{Nk}_{\mathrm{s}}}\right)}
$$

As discussed in the text, in most practical applications, one expects the force to be well bel ow the scission force, which leads to $f_{n} \ll k_{s}$. Using this, we can expand the denominator to obtain

$$
\bar{F}=\frac{1}{2 N k_{s} n=1} \sum_{n}^{N} f_{n}^{2}\left(1-\frac{1}{N k_{s}} \sum_{=1}^{N} \mathrm{mf}_{m}\right)
$$

Averaging now over the PDF of $f$ gives

$$
\begin{aligned}
\bar{F}=\frac{(N-1)}{4 k_{s}}\left\langle f^{2}\right\rangle- & \frac{1}{4 k_{s}^{2}}\left[\frac{(N-1)^{2}}{2}\left\langle f^{2}\right\rangle\langle f\rangle+\right. \\
& \left.\frac{(N+1)(2 N+1)}{3 N}\left(\left\langle f^{3}\right\rangle-\left\langle f^{2}\right\rangle\langle f\rangle\right)\right]
\end{aligned}
$$

\section{References and Notes}

(1) For example, see: Evans, E.; Ritchie, K. Biophys. J . 1997, 72, 1541-1555. Grandbois, M.; Beyer, M.; Rief, M.; C-Schaumann, H.; Gaub, H. E. Science 1999, 283, 1727-1730. Bemis, J . E.; Akhremitchev, B. B.; Walker, G. C. Langmuir 1999, 15, 2799-3805.

(2) Edwards, S. F. Proc. Phys. Soc. 1967, 92, 9-16. Graessley, W. W. Adv. Polym. Sci. 1982, 47, 67-117. Doi, M.; Edwards, S. F. The Theory of Polymer Dynamics; Clarendon Press: Oxford, 1986.

(3) For example, see: Kramer, O. British Polym. J . 1985, 17, 129-133. Plummer, C. J .; Donald, A. M. Macromolecules 1990, 23, 3929-3937. Creton, C.; Kramer, E. J .; Hui, C. Y.; Brown, H. R. Macromolecules 1992, 25, 3075-3088. Han, H. Z. Y.; MCLeish, T. C. B.; Duckett, R. A.; Ward, N.J .; J ohnson, A. F.; Donald, A. M.; Butler, M. Macromolecules 1998, 31, 1348-1357.

(4) Blumenfeld, R. Disentangl ement Dynamics and Applications to Deformations in Polymer Glasses and Interfaces, in preparation.

(5) Flory, P. J . Principles of Polymer Chemistry; Cornell University Press: Ithaca, NY, 1953.

(6) Angles larger than $2 \pi$ are irrelevant here: First, their probability of occurrence is small; second, in this case, the chain would not disentangle by simple pulling.

(7) Flory, P. J . Statistical Mechanics of Chain Molecules; WileyInterscience: New York, 1969. Treloar, L. R. G. Physics of Rubber Elasticity, 3rd ed.; Oxford University Press: London, 1975.

(8) Blumenfeld, R. Hysteretic Force Response in Single Chain Pullout, in preparation.

MA9912251 